\title{
Ý ĐỊNH SỬ DỤNG ỨNG DỤNG DI ĐộNG ĐỂ LỰA CHỌN CÁC DICCH VỤ KHI DU LỊCH CỦA GIỚI TRẺ TRONG THỜI KỲ CHUYỂN ĐỔI SỐ
}

\author{
BÙI THÀNH KHOA, TRÀ̀ THI HUÊ CHI, MAI THANH HÙNG, VŨ THỊ MAI CHI \\ Truờng Đại học Công nghiệp thành phố Hồ Chi Minh \\ buithanhkhoa@iuh.edu.vn
}

Tóm tắt. Sự phát triển của công nghệ đã thay đổi các hoạt động trong cuộc sống của con người, bao gồm cả hoạt động du lịch. Các du khách trẻ tuổi là những người có sự hiểu biết về công nghệ và thường xuyên đi du lịch, nên việc lựa chọn các dịch vụ khi du lịch là điều không thể tránh khỏi. Dựa trên mô hình chấp nhận công nghệ, mục tiêu của nghiên cứu này nhằm tìm hiểu về ý định sử dụng các ứng dụng di động để lựa chọn các dịch vụ du lịch của thế hệ $\mathrm{Z}$ trong thời kỳ chuyển đổi số. Bên cạnh phương pháp nghiên cứu định tính để xác nhận và điều chỉnh thang đo, nghiên cứu còn sử dụng phương pháp nghiên cứu định lượng thông qua việc sử dụng bảng câu hỏi trực tuyến tự quản trị để khảo sát 786 du khách thuộc thế hệ $Z$, những người từng đi du lịch và sử dụng các ứng dụng di động để đặt các dịch vụ trong quá trình thực hiện chuyến đi. Kết quả nghiên cứu ngoài khẳng định lại kết quả của mô hình chấp nhận công nghệ (TAM) được đề xuất bởi Davis (1986), thì còn chỉ ra rằng tính cách đổi mới của du khách là yếu tố bên ngoài ảnh hưởng tích cực đến quá trình chấp nhận lựa chọn khi đi du lịch của du khách thế hệ Z. Nghiên cứu cũng đề xuất một số giải pháp cho các doanh nghiệp kinh doanh dịch vụ liên quan đến du lịch khi xây dựng, hoặc sử dụng các ứng dụng di động để quảng bá.

Từ khóa. Tính cách tân, mô hình TAM, ứng dụng di động, chuyển đổi số, dịch vụ du lịch, thế hệ Z.

\section{BEHAVIORAL INTENTION TO USE THE MOBILE APPLICATIONS FOR GETTING THE TOURISM SERVICES OF THE YOUTH PEOPLE IN DIGITAL TRANSFORMATION ERA}

\begin{abstract}
The advancement of technology has changed activities in people's lives, including tourism. Young travelers are technology savvy and often travel, so getting these tourism services is inevitable. Based on the technology adoption model, the goal of this study is to learn about the intention of using mobile applications to select Generation Z's tourism services in the digital transformation era. In addition to qualitative research methods to confirm and adjust the scale, the research also uses quantitative research methods through the online self-administered questionnaire to survey 786 visitors of the generation $\mathrm{Z}$ who have traveled and used mobile applications to get the services during their trip. The research results, in addition to confirming the results of the Technology Acceptance Model (TAM) proposed by Davis (1986), also showed that the innovation of the visitor is an external factor that positively affects the acceptance of the mobile app of generation $\mathrm{Z}$ in tourism. The study also recommends several solutions for tourism-related service businesses when building or using mobile apps to advertise.
\end{abstract}

Keywords. Innovation, TAM model, mobile applications, digital transformation, tourism services, Z Generation.

\section{GIÓ́I THIỆ}

Công nghệ đang làm thay đổi một cách nhanh chóng các hoạt động của đời sống như giáo dục, ngân hàng, thương mại, nghiên cứu và cả ngành du lịch (Agag \& El-Masry, 2016; Hoa \& Khoa, 2021; Khoa và cộng $s u ̛$, 2020; Santini và công sư, 2020). Các ứng dụng di động chủ đề về du lịch đứng thứ 7 trong nhóm được tải về nhiều trên kho ứng dụng; và hiện có khoảng $30 \%$ số người dùng các ứng dụng di động để tìm kiếm giá máy bay hoặc phòng giá rẻ, $8 \%$ khách hàng dựa hoàn toàn vào ứng dụng di động để lên lịch trình và đặt vé (Vipin, 2016). Sự bùng nổ của tiện ích di động đã tác động mạnh đến ngành công nghiệp du lịch, giúp cho quá trình đặt vé máy bay, hoặc đặt phòng khách sạn trở nên dễ dàng hơn. Ngày nay, du khách có thể sử dụng Internet kết hợp với các ứng dụng di động được cài đặt các thiết bị thông minh để tìm kiếm thông tin du lịch, tham khảo điểm đến, so sánh và lựa chọn các dịch vụ du lịch hợp lý, thực hiện các giao 
dịch mua tour, đặt phòng, mua vé máy bay, thanh toán trực tuyến. Điều này chứng tỏ tầm quan trọng của ứng dụng công nghệ thông tin trong phát triển của du lịch.

Hệ thống phân phối toàn cầu (Global Distribution System - GDS) đã trở thành lựa chọn cho ngành Du lịch, vì hệ thống có thể thay đổi các yêu cầu của khách du lịch về các hãng hàng không và các công ty du lịch lữ hành, nhà ở, đến các siêu thị du lịch điện tử và các đơn vị kinh doanh chiến lược (Radulović, 2013). GDS ngày càng cung cấp cả sản phẩm giải trí và kinh doanh, bằng cách cung cấp thông tin và cho phép đặt chỗ, gói du lịch và các điểm du lịch. Do đó, việc các doanh nghiệp du lịch - lữ hành ứng dụng công nghệ thông tin vào việc tiếp cận với khách hàng ngày càng phổ biến, qua đó đưa chiến lược công nghệ thông tin hóa trở thành chiến lược không thể thiếu của các doanh nghiệp trong thời kỳ chuyển đổi số. Tuy nhiên, việc sử dụng công nghệ để lựa chọn các dịch vụ trong du lịch không phải được chấp nhận bởi toàn bộ các khách hàng, mặc dù các lợi ích của công nghệ mang lại là rõ ràng. Nhiều khách hàng vẫn còn lo ngại và chưa thực sự mạnh dạn trong việc sử dụng các thiết bị di động để đặt các dịch vụ khi du lịch, ngoại trừ các du khách trẻ tuổi, ở thế hệ Y và thế hệ Z. Đối với Thế hệ Z (sinh sau năm 1995), chiếm 32\% trong dân số toàn cầu, vượt qua Thiên niên kỷ $(31,5 \%)$ cho vị trí hàng đầu về số lượng (Lee \& Wei, 2018). Những du khách thế hệ $Z$ có ý thức về nhận thức toàn cầu, họ di chuyển thoải mái xuyên biên giới và trên hết, họ coi du lịch là ưu tiên hàng đầu. $65 \%$ du khách thuộc Thế hệ $Z$ xếp hạng "du lịch và ngắm nhìn thế giới" là cách quan trọng nhất để sử dụng tiền của họ (World Tourism Organization, 2016). Mặc dù có ý thức về ngân sách, thế hệ Z sẽ chi tiêu nhiều hơn cho những chuyến du lịch trải nghiệm, cho phép họ khám phá cuộc sống như một người dân địa phương chứ không phải như một khách du lịch.

Một trong những lý do khiến thế hệ $Z$ tự tin khi đi du lịch đó là khả năng sử dụng công nghệ. Sự phát triển của công nghệ đã tạo nhiều điều kiện cho những thế hệ có tư tưởng cách tân, có nghĩa là những người muốn tìm hiểu những điều mới, bao gồm kể cả công nghệ mới. Internet chính thức được thương mại hóa vào năm 1996, do đó, thế hệ $Z$ lớn lên trong thời kỳ tiến bộ công nghệ thay đổi nhanh và mạnh nhất trong lịch sử loài người. Trên thực tế, thế hệ $Z$ dành trung bình 3 giờ 38 phút trực tuyến trên điện thoại thông minh, lâu hơn gần 50 phút so với người dùng Internet trung bình (GlobalWeb Index, 2016). Việc có quyền truy cập 24/7 vào thông tin trên toàn thế giới thông qua một thiết bị di động giúp cho Thế hệ $Z$ giải quyết vấn đề, mạng, giao tiếp, học hỏi, mua hàng và cuối cùng là cách họ thể hiện ở nơi làm việc. Rogers (1962) cũng đã từng đánh giá những người cách tân (Innovators) và sớm chấp nhận công nghệ (Early adoptors) là những đối tượng tiên phong chấp nhận sử dụng công nghệ mới, dù biết nó có thể mang lại rủi ro. Do đó, việc chấp nhận một công nghệ xuất phát không chỉ từ bản thân công nghệ đó, chẳng hạn từ tính dễ dùng và tính hữu dụng, mà còn từ bản thân người dùng, chẳng hạn, tính cách cách tân. Davis (1989) cũng từng cho rằng, những yếu tố bên ngoài (External factors) có ảnh hưởng tích cực đến quá trình chấp nhận công nghệ của người sử dụng và đề xuất đây là hạn chế của mô hình chấp nhận công nghệ. Vì lý do trên, nghiên cứu ý định sử dụng ứng dụng di động để lựa chọn các dịch vụ khi du lịch của giới trẻ trong thời kỳ chuyển đổi số sẽ mang lại cả ý nghĩa về mặt lý luận và cả thực tiễn đối với các doanh nghiệp kinh doanh dịch vụ du lịch, lữ hành. Mục tiêu nghiên cứu này nhằm:

- Khẳng định lại mối quan hệ giữa các yếu tố trong mô hình chấp nhận công nghệ TAM của Davis (1989)

- Đánh giá ảnh hưởng của tính cách tân người dùng đối với phương diện cảm nhận tính hữu ích và cảm nhận dễ sử dụng của ứng dụng di động trong du lịch

- Đề xuất một số hàm ý quản trị cho các doanh nghiệp ngành du lịch để nâng cao hiệu quả kinh doanh Phần tiếp theo của nghiên cứu sẽ trình bày cơ sở lý thuyết cũng như xây dựng các giả thuyết nghiên cứu. Sau đó, nghiên cứu sẽ chỉ rõ phương pháp nghiên cứu đã được thực hiện nhằm đạt được mục tiêu nghiên cứu. Kết quả nghiên cứu và thảo luận là một trong những phần quan trọng của nghiên cứu này. Cuối cùng, nghiên cứu cũng nêu lên các hạn chế của nghiên cứu để làm tiền đề cho các nghiên cứu tiếp theo.

\section{CƠ SỞ LÝ THUYẾT}

\subsection{Mô hình nghiên cứu đề xuất}

Mô hình chấp nhận công nghệ TAM được nhiều nhà nghiên cứu áp dụng ở nhiều lĩnh vực như chấp nhận sử dụng máy vi tính (Dinev và cộng sư, 2009), thương mại điện tử (Featherman \& Fuller, 2003), thương mại di động (Alsamydai, 2014), giáo dục (Khoa và cộng sụ, 2020). Mặc dù TAM đã bị chỉ trích ở một số khía cạnh, nhưng vẫn được coi là một khung lý thuyết chung hữu ích và phù hợp với một số cuộc điều tra liên quan đến các yếu tố ảnh hưởng đến ý định sử dụng công nghệ mới của người dùng (Braun, 2019). Dựa 
trên TAM, một số nghiên cứu đã chứng minh rằng cảm nhận hữu ích và cảm nhận tính dễ sử dụng là hai khía cạnh khác nhau của một công nghệ tác động đến nhận thức và hành vi của người sử dụng hệ thống (Khoa và công sư, 2020). Mô hình Chấp nhận công nghệ TAM cho rằng việc sử dụng hệ thống thông tin là kết quả của ý định sử dụng công nghệ, nhưng mặt khác, ý định này sẽ bị chi phối bởi thái độ của người đó đối với việc sử dụng hệ thống và cũng bởi nhận thức của người dùng về sự hữu ích. Theo Davis (1986), thái độ của một cá nhân không phải là yếu tố duy nhất quyết định việc họ sử dụng một hệ thống, mà còn dựa trên tác động của nó đối với hiệu suất sử dụng công nghệ. Do đó, ngay cả khi người dùng không hoan nghênh một hệ thống thông tin, khả năng họ vẫn sẽ sử dụng công nghệ đó là rất cao nếu nhận thức rằng hệ thống đó sẽ cải thiện hiệu suất trong công việc. Bên cạnh đó, mô hình chấp nhận công nghệ đưa ra giả thuyết về mối liên hệ trực tiếp giữa tính hữu ích và tính dễ sử dụng.

Nhận thức tính dễ sử dụng ảnh hưởng một cách đáng kể đến thái độ của một cá nhân thông qua hai cơ chế chính, bao gồm hiệu quả bản thân và tính công cụ (Davis, 1986). Hiệu quả bản thân là một khái niệm giải thích rằng một hệ thống càng dễ sử dụng, thì cảm giác hiệu quả của người dùng càng lớn (Bandura, 1982). Hơn nữa, một công cụ dễ sử dụng sẽ khiển người dùng cảm thấy rằng họ có quyền kiểm soát những gì mình đang làm. Hiệu quả là một trong những yếu tố chính tạo nên động lực nội tại (Bandura, 1982) và tạo ra mối liên hệ trực tiếp giữa tính dễ sử dụng và thái độ. Tính dễ sử dụng cũng có thể đóng góp một cách cụ thể vào việc cải thiện hiệu suất của một người. Do thực tế là người dùng sẽ phải triển khai ít nỗ lực hơn với một công cụ dễ sử dụng, nên họ sẽ có thể dành những nỗ lực để hoàn thành các nhiệm vụ khác (Davis, 1986). Ngoài ra, nhiều nhà nghiên cứu của chỉ ra hạn chế của TAM, trong đó có cả tác giả của mô hình. Davis và cộng sư (1989) đã chỉ ra rằng cần có những mở rộng để tạo ra sự hiểu biết sâu sắc về sự chấp nhận sử dụng một công nghệ ngoài các yếu tố thuộc về công nghệ như tính hữu dụng và tính dễ sử dụng của công nghệ. Một số nghiên cứu đã chỉ ra những bất cập của TAM trong việc giải quyết mối liên hệ giữa công nghệ và việc áp dụng công nghệ trên thực tế. Bằng chứng đầy đủ từ các nghiên cứu liên quan đến mô hình TAM đã chỉ ra rằng TAM không thể cung cấp các tiền đề toàn diện cho việc sử dụng thiết bị di động, hoặc ảnh hưởng xã hội và tạo điều kiện cho hành vi (Napitupulu, 2017; Torres \& Gerhart, 2017). Lập luận chỉ ra rằng, mặc dù phổ biến tuy nhiên mô hình TAM không phù hợp để giải thích việc người dùng chấp nhận và sử dụng công nghệ mới, đặc biệt là trong bối cảnh chính phủ điện tử (Chandio và cộng sự, 2017). Do đó, việc nghiên cứu các yếu tố bên ngoài cụ thể là quan trọng để hoàn thiện mô hình TAM. Liébana-Cabanillas và cộng sư (2020) đã chỉ ra rằng tính cách tân của người dùng sẽ là động cơ cho sự chấp nhận sử dụng phương thức thanh toán qua di động. Trong lý thuyết khuyến tán đổi mới, Rogers (1962) chỉ ra tính cách tân là yếu tố sẽ dẫn đến hành vi sử dụng những công nghệ của người dùng. Do đó, tính cách tân có thể là yếu tố đầu vào quan trọng cho sự đánh giá việc chấp nhận một công nghệ mới, chẳng hạn như ý định sử dụng ứng dụng di động để lựa chọn các dịch vụ khi du lịch của giới trẻ trong thời kỳ chuyển đổi số. Dựa trên những lập luận trên, nghiên cứu đề xuất mổ hình nghiên cứu như sau:

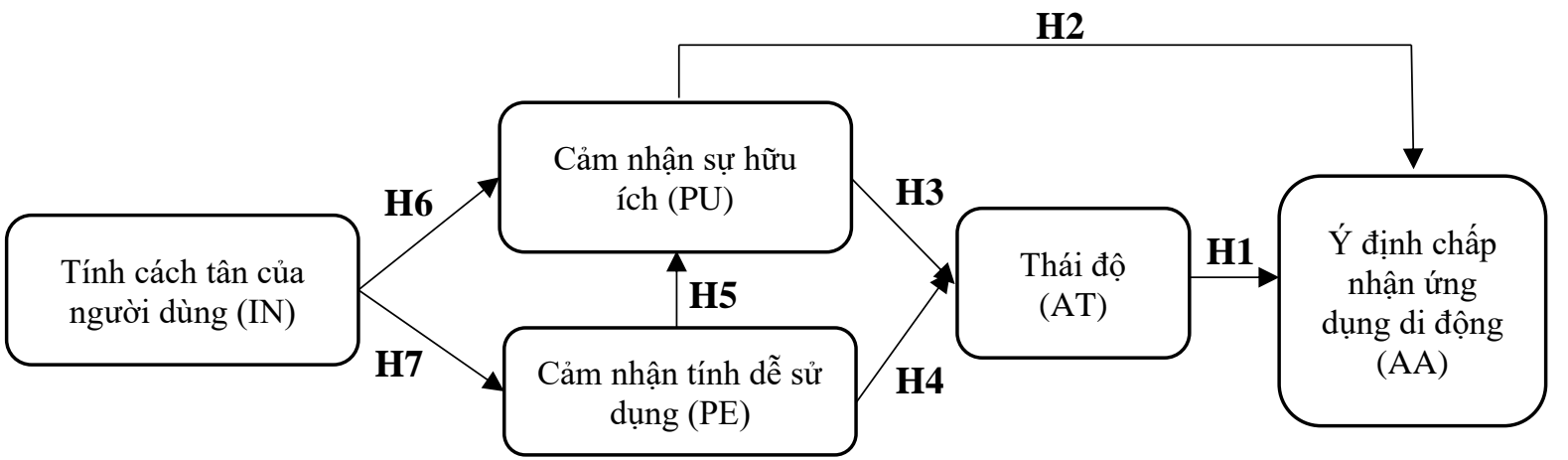

\subsection{Giả thuyết nghiên cứu}

Hình 1: Mô hình nghiên cứu

Dựa trên lý thuyết của mô hình TAM, ý định sử dụng ứng dụng di động để đặt các dịch vụ khi đi du lịch của du khách thế hệ $Z$ sẽ phụ thuộc vào thái độ và cảm nhận sự hữu ích đối với việc sử dụng ứng dụng di động để thực hiện các hoạt động liên quan đến chuyến đi của du khách. Nhiều nghiên cứu cũng đã chỉ ra rằng, người dùng hài lòng hoặc thích một công nghệ nào đó sẽ dễ dẫn đến hành vi sử dụng công nghệ đó cho các hoạt động trong đời sống (Alsamydai, 2014; Lee và công sụ, 2011; Syed-Abdul và cộng sự, 2019). 
Hoặc ngược lại, khi người dùng lo sợ một công nghệ, thì có thể sẽ dẫn đến quyết định không sử dụng công nghệ đó trong cuộc sống. Đôi khi, khách hàng cũng có thể đánh đổi để có thể nhận được các dịch vụ tốt nếu họ nhận thấy sự đánh đổi là hợp lý (Khoa, 2020a; Khoa \& Nguyễn, 2020). Khi các du khách có thái độ tốt đối với việc sử dụng điện thoại di động cho hoạt động tìm kiếm, lựa chọn dịch vụ khi đi du lịch; họ sẽ sẵn sàng sử dụng các ứng dụng liên quan đến du lịch. Giả thuyết $\mathrm{H} 1$ được đề xuất như sau:

H1: Thái độ đối với ưng dụng di động ảnh hưởng tích cực đến ý định sủ dụng ứng dụng di động để lựa chọn các dịch vu khi du lịch của giới trẻ trong thời kỳ chuyển đổi số.

Ngoài ra, khi một công nghệ mang lại lợi ích cho người sử dụng như sự thuận tiện, tiết kiệm thời gian, giảm các nỗ lực nhưng vẫn đạt hiệu quả mong muốn sẽ khiến cho người dùng mong muốn sử dụng công nghệ (Munoz-Leiva và cộng sư, 2017; Syed-Abdul và cộng sụ, 2019). Tính hữu ích được định nghĩa là mức độ mà một người tin rằng việc sử dụng một hệ thống sẽ cải thiện hiệu suất của mình (Luan \& Teo, 2009). Úng dụng công nghệ, cụ thể là sử dụng ứng dụng di động để tham khảo thông tin, sẽ giúp du khách thế hệ $Z$ dễ dàng chọn lựa đúng những dịch vụ mà bản thân cần cho chuyến đi. Ngoài ra, việc sử dụng ứng dụng để đặt trước các dịch vụ sẽ giúp cho du khách đỡ tốn thời gian tìm kiếm khi đến địa điểm du lịch lạ. Do đó, những lợi ích này sẽ thúc đẩy du khách sử dụng một cách tích cực ứng dụng liên quan cho hoạt động du lịch. Từ đó, nghiên cứu đề xuất giả thuyết $\mathrm{H} 2$ :

H2: Cảm nhận sụ hũu ích của ứng dụng di động ảnh hưởng tích cực đến ý định sử dụng úng dụng di động để lựa chọn các dịch vu khi du lịch của giới trẻ trong thời kỳ chuyển đổi số.

Tính hữu ích và tính dễ sử dụng của công nghệ là các yếu tố quan trọng, ảnh hưởng đến ý thái độ của người dùng đối với công nghệ (Davis, 1986; Davis, 1989). Mức độ dễ sử dụng là mức độ mà một người tin rằng việc sử dụng một hệ thống sẽ dễ dàng, ít tốn công sức để đạt được mục tiêu (Arif và công sư, 2016). Thay vì phải đến phòng vé để mua vé phương tiện vận tải, hoặc phải đến tận nơi mới có thể biết về chất lượng dịch vụ, các du khách thế hệ $Z$ có thể thực hiện các hoạt động đó nhanh chóng thông qua ứng dụng di động. Điều này khiến cho khách hàng cảm thấy thích thú, và nhận ra rằng sử dụng công nghệ là cần thiết cho chuyến du lịch của họ (Agag \& El-Masry, 2016). Do đó, giả thuyết H3 được đề xuất như sau:

H3: Cảm nhận sụ hữu ich của ứng dụng di động ảnh hương tich cực thái độ đối với ưng dụng di động khi lự chọn các dịch vu khi du lịch của giới trẻ trong thời kỳ chuyển đổi số.

Một ảnh hưởng tương tự đến thái độ của người dùng, đó chính là tính dễ sử dụng. Người dùng sẽ cảm thấy không thoải mái hay thậm chí có thể khó chịu và ghét một công nghệ khi công nghệ đó quá phức tạp để sử dụng hay quá khó để học cách sử dụng (Munoz-Leiva và cộng sự, 2017). Một ứng dụng đặt chỗ trong du lịch nếu có đa ngôn ngữ, hướng dẫn sử dụng rõ ràng sẽ khiến du khách thoải mái, và thích sử dụng nó cho hoạt động đặt chuyến du lịch, hay khách sạn. Ngoài ra, tính dễ sử dụng được xem như một tiền đề tạo ra cảm nhận hữu ích của công nghệ (Chuttur, 2009; Lin và cộng sự, 2014). Nếu du khách phải tốn quá nhiều thời gian mới biết cách sử dụng ứng dụng, hay tốn nhiều công sức mới mua được vé qua ứng dụng thay vì ra quầy vé, họ sẽ xem ứng dụng đó là vô ích. Do đó, giả thuyết $\mathrm{H} 4$ và giả thuyết $\mathrm{H} 5$ được đề xuất:

H4: Cảm nhận tính dễ sủ dụng của ưng dụng di động ảnh hưởng tích cực thái độ đối với ứng dụng di động khi lự chọn các dịch vu khi du lịch của giới trẻ trong thời kỳ chuyển đổi số.

H5: Cảm nhận tính dễ sử dụng ảnh hương tích cực đến cảm nhận sự hữu ich của ứng dụng di động khi lựa chon các dịch vu khi du lịch của giới trẻ trong thời kỳ chuyển đổi số.

Đổi mới là một phạm trù rộng, liên quan đến kiến thức hiện tại của đơn vị được phân tích. Bất kỳ ý tưởng, thực hành hoặc đối tượng nào được một cá nhân hoặc đơn vị khác chấp nhận đều có thể được coi là một sự đổi mới có sẵn để nghiên cứu (Khoa, 2020b). Agarwal (2000) cũng đã chỉ ra rằng người dùng tiềm năng đưa ra quyết định áp dụng hoặc từ chối một sự đổi mới dựa trên niềm tin mà họ hình thành về đổi mới đó. Những cá nhân, hoặc tổ chức có tính cách tân sẽ dễ dàng chấp nhận sự đổi mới, mặc dù có thể khó khăn hay sự rủi ro trong thời gian đầu (Al-Rahmi và cộng sự, 2019). Những người cách tẩn thường sẽ cảm thấy rằng công nghệ đổi mới sẽ mang lại cho họ hiệu quả công việc cao hơn và từ đó họ cảm nhận được tính có ích của công nghệ đó (Taib và cộng sư, 2016). Thế hệ Z là những người dùng công nghệ, họ sinh ra trong thời khoa học kỹ thuật phát triển nhanh chóng sau khi có Internet; do đó, khả năng thích ứng với công nghệ mới, cũng như tìm hiểu về công nghệ mới của thế hệ $Z$ được đánh giá là tốt. Họ chủ động tìm kiếm và dể dàng tìm thấy các hướng dẫn sử dụng từ Google hay các website, nên việc sử dụng công nghệ, ứng dụng trở nên dễ dàng hơn rất nhiều (Ayar và cộng sự, 2019). Từ đó, nghiên cứu đề xuất giả thuyết: 
H6: Tính cách tân của người dùng ảnh hưởng tích cực đến cảm nhận sự hữu ích của ứng dụng di động khi lự chọn các dịch vu khi du lịch của giới trẻ trong thời kỳ chuyển đổi số.

H7: Tính cách tân của người dùng ảnh hưởng tích cực đến cảm nhận tính dễ sủ̉ dụng của ứng dụng di động khi lụa chọn các dịch vu khi du lịch của giới trẻ trong thời kỳ chuyển đổi số.

\section{PHƯONG PHÁP NGHIÊN CÚU}

Phương pháp nghiên cứu hỗn hợp được sử dụng trong nghiên cứu này. Đầu tiên, nghiên cứu định tính được sử dụng nhằm mục đích xác nhận các cấu trúc nghiên cứu cũng như điều chỉnh các chỉ mục nghiên cứu. Phương pháp thu thập dữ liệu định tính là phương pháp thảo luận nhóm. Thông qua phương pháp chọn mẫu phát triển mầm, buổi thảo luận nhóm được thực hiện với sự tham gia của 9 chuyên gia, bao gồm 04 giảng viên đại học ngành công nghệ thông tin, và du lịch; 03 giám đốc, phó giám đốc các công ty du lịch, lữ hành; 02 khách hàng thường xuyên du lịch và đã có kinh nghiệm sử dụng ứng dụng di động để đặt các dịch vụ khi đi du lịch. Cuộc thảo luận diễn ra trong 90 phút, tại phòng nghiên cứu của trường Đại học Công nghiệp Thành phố Hồ Chí Minh. Kết quả của thảo luận nhóm đó là sự đồng tình về các yếu tố nghiên cứu, cũng như điều chỉnh các thang đo dựa trên thang đo gốc, nhằm phục vụ cho giai đoạn nghiên cứu định lượng tiếp theo.

Bảng 1: Thông tin mẫu nghiên cứu

\begin{tabular}{|c|c|c|c|}
\hline & & $\mathbf{N}$ & Phần trăm (\%) \\
\hline \multirow{2}{*}{ Giới tính } & Nam & 409 & 52.0 \\
\hline & Nũ & 377 & 48.0 \\
\hline \multirow{3}{*}{ Độ tuổi } & $<18$ tuổi & 134 & 17.1 \\
\hline & $18-22$ & 302 & 38.4 \\
\hline & $23-25$ & 350 & 44.5 \\
\hline \multirow{4}{*}{$\begin{array}{l}\text { Nghề } \\
\text { nghiệp }\end{array}$} & Học sinh & 133 & 16.9 \\
\hline & Sinh viên & 202 & 25.7 \\
\hline & Giảng viên & 191 & 24.3 \\
\hline & Nhân viên văn phòng & 260 & 33.1 \\
\hline \multirow{3}{*}{ Khu vực } & Hà Nội & 159 & 20.2 \\
\hline & Thành phố Hồ Chí Minh & 354 & 45.0 \\
\hline & Đà Nẵng & 273 & 34.7 \\
\hline
\end{tabular}

Các thang đo trong nghiên cứu chủ yếu được kế thừa và phát triển từ các nghiên cứu trước đây. Tính cách tân của người dùng (IN) đo lường với bốn chỉ mục, và được đề xuất bởi Ramos de Luna và cộng sụ (2020). Các thang đo trong mô hình TAM như cảm nhận sự hữu ích, cảm nhận tính dễ dùng, và ý định sử dụng ứng dụng di động để lựa chọn các dịch vụ khi đi du lịch được điều chỉnh từ các thang đo trong nghiên cứu của Venkatesh và Davis (2000), chẳng hạn, cảm nhận sự hữu ích $(\mathbf{P U})$ với 4 chỉ mục, cảm nhận tính dễ dùng (PE) với 4 chỉ mục, và ý định sử dụng ứng dụng di động để lựa chọn các dịch vụ khi đi du lịch $(\mathbf{A A})$ với 2 chỉ mục. Cuối cùng, thái độ đối với ứng dụng di động $(\mathbf{A T})$, bao gồm 4 chỉ mục và được kế thừa từ nghiên cứu của Zhang và cộng sự (2008). Nghiên cứu định lượng sẽ được thực hiện để kiểm định các giả thuyết và mô hình nghiên cứu. Một bảng câu hỏi tự quản lý trực tuyến được sử dụng để khảo sát 786 du khách thuộc thế hệ Z tại các thành phố lớn là thành phố Hồ Chí Minh, thành phố Hà Nội, thành phố Đà Nẵng. Phương pháp chọn mẫu trong nghiên cứu định lượng là phương pháp chọn mẫu theo mục đích. Đáp viên là những người đã từng đi du lịch, quan tâm và có nhu cầu sử dụng các thiết bị di động để đặt các dịch vụ khi có nhu cầu du lịch. Thông tin đáp viên được trình bày trong bảng 1 . Dữ liệu thu thập sau khi được sàng lọc được xử lý bằng phần mềm SPSS 26 và SmartPLS 3.2.7.

\section{KẾT QUẢ NGHIÊN CÚU}

Nghiên cứu sử dụng quy trình kiểm định của Hair và cộng sự (2017) để kiểm định các giả thuyết và mô hình cấu trúc tuyến tính được đề xuất theo quy trình đánh giá mô hình đo lường, sau đó sẽ đánh giá mô hình cấu trúc tuyến tính. 


\section{1 Đánh giá mô hình đo lường}

Đầu tiên, nghiên cứu sẽ kiểm định độ tin cậy và độ giá trị của các thang đo. Hệ số Cronbach's Alpha $(\mathrm{CA})$ của các thang đo nghiên cứu được đề xuất là lớn hơn 0,7 để đạt được độ tin cậy nội tại (Nunnally \& Bernstein, 1994). Ngoài ra, việc đánh giá độ giá trị bao gồm đánh giá về độ giá trị phân biệt và độ giá trị hội tụ. Giá trị phân biệt được đánh giá thông qua hệ số HTMT, với tiêu chuẩn là HTMT của cặp cấu trúc sẽ nhỏ hơn 0,85. Giá trị hội tụ của một thang đo được đánh giá thông qua hệ số tải ngoài (outer loading $>=$ $0,708$ ), độ tin cậy tổng hợp (Composite Reliability, $C R>=0,7)$, và phương sai trích trung bình (Average Variance Extracted, AVE >= 0,5) (Hair và công sư, 2017).

Kết quả của bảng 2 đã chỉ ra các thang đo đều đạt được độ tin cậy nội tại, với toàn bộ $\mathrm{CA}>0,7$. Ngoài ra, hệ số $\mathrm{CR}$ của tất cả cấu trúc cũng lớn hơn 0,7 ; $\mathrm{AVE}$ lớn hơn 0,5 ; và hệ số tải ngoài cũng lớn hơn 0,708 ; do đó, các cấu trúc đều đạt được giá trị hội tụ. Đồng thời, các cấu trúc cũng phân biệt với nhau, với HTMT giữa các cấu trúc đều nhỏ hơn 0,85 .

\section{2 Đánh giá mô hình cấu trúc}

Tiếp theo, nghiên cứu sẽ đánh giá mô hình nghiên cứu và các giả thuyết nghiên cứu. Theo đề xuất của Hair và cộng sự (2017), nghiên cứu sẽ đánh giá sự cộng tuyến, các hệ số đường dẫn mô hình cấu trúc, hệ số xác định (giá trị $\left.\mathrm{R}^{2}\right)$, hệ số tác động $\left(\mathrm{f}^{2}\right)$, và sự liên quan dự báo $\left(\mathrm{Q}^{2}\right)$.

Đa cộng tuyến (Multicollinearity) là hiện tượng thường xảy ra khi mối tương quan cao giữa hai hay nhiều biến độc lập trong mô hình hồi quy. Nói cách khác, một biến độc lập có thể sử dụng để dự đoán một biến độc lập khác. Để nhận biết hiện tượng đa cộng tuyến, ta có thể áp dụng một thử nghiệm rất đơn giản đó chính là dựa vào hệ số phóng đại phương sai VIF (Variance inflation factor) để xác định mối tương quan giữa các biến độc lập và sức mạnh của mối tương quan đó. Giá trị VIF bắt đầu từ 1 và không có giới hạn trên. Giá trị VIF trong khoảng từ [1-2] sẽ chỉ ra rằng không có mối tương quan giữa biến độc lập này và bất kỳ biến nào khác. Do đó, kết quả của bảng 3 đã chỉ ra rằng không có hiện tượng đa cộng tuyến giữa các biến độc lập trong nghiên cứu này, khi toàn bộ hệ số VIF đều nhỏ hơn 2.

Bảng 2: Kết quả đánh giá mô hình đo lường trong nghiên cứu

\begin{tabular}{|l|c|c|c|c|c|c|c|c|c|}
\hline \multirow{2}{*}{ Cấu trúc } & \multirow{2}{*}{ CA } & \multirow{2}{*}{ CR } & \multirow{2}{*}{ AVE } & & \multicolumn{2}{|c|}{ Hệ số tải ngoài } & \multicolumn{4}{|c|}{ HTMT } \\
\cline { 5 - 11 } & & & Nhỏ nhất & Lớn nhất & AA & AT & IN & PE \\
\hline AA & 0,876 & 0,942 & 0,890 & 0,942 & 0,944 & & & & \\
\hline AT & 0,818 & 0,880 & 0,648 & 0,749 & 0,865 & 0,579 & & & \\
\hline IN & 0,835 & 0,889 & 0,668 & 0,775 & 0,835 & 0,461 & 0,536 & & \\
\hline PE & 0,899 & 0,930 & 0,769 & 0,854 & 0,917 & 0,447 & 0,662 & 0,532 & \\
\hline PU & 0,819 & 0,882 & 0,655 & 0,768 & 0,886 & 0,738 & 0,807 & 0,690 & 0,645 \\
\hline
\end{tabular}

Bảng 3: Hệ số VIF

\begin{tabular}{|l|r|r|l|l|l|}
\hline & \multicolumn{1}{|l|}{ AA } & AT & IN & PE & PU \\
\hline AA & & & & & \\
\hline AT & 1,756 & & & & \\
\hline IN & & & & 1,000 & 1,279 \\
\hline PE & & 1,438 & & & 1,279 \\
\hline PU & 1,756 & 1,438 & & & \\
\hline
\end{tabular}

Ngoài ra, hệ số xác định (coefficient of determination) thường ký hiệu là $\mathrm{R}^{2}$, một con số thống kê tổng hợp khả năng giải thích của một phương trình. $\mathrm{R}^{2}$ biểu thị tỷ lệ biển thiên của biến phụ thuộc do tổng mức biến thiên của các biến giải thích gây ra. Đối với khoa học hành vi, $\mathrm{R}^{2}$ lớn hơn $20 \%$ được xem như là cao (Hair và cộng sự, 2017).

Bảng 4: Giá trị $\mathrm{R}^{2}, \mathrm{f}^{2}$, và $\mathrm{Q}^{2}$

\begin{tabular}{|c|c|c|c|c|c|c|}
\hline \multirow{2}{*}{ Cấu trúc } & \multirow{2}{*}{$\mathrm{R}^{2}$} & \multicolumn{4}{|c|}{$\mathrm{f}^{2}$} & \multirow{2}{*}{$\mathrm{Q}^{2}$} \\
\hline & & $\mathrm{AA}$ & $\mathrm{AT}$ & $\mathrm{PE}$ & $\mathrm{PU}$ & \\
\hline AA & 0,401 & & & & & 0,354 \\
\hline AT & 0,496 & 0,02 & & & & 0,315 \\
\hline IN & & & & 0,279 & 0,229 & \\
\hline PE & 0,218 & & 0,13 & & 0,181 & 0,165 \\
\hline PU & 0,434 & 0,266 & 0,327 & & & 0,278 \\
\hline
\end{tabular}


Với $\mathrm{R}_{\mathrm{AA}}^{2}=0,401, \mathrm{R}_{\mathrm{AT}}^{2}=0,496, \mathrm{R}_{\mathrm{PE}}^{2}=0,218$, và $\mathrm{R}_{\mathrm{PU}}^{2}=0,434 ; \mathrm{R}^{2}$ của các biến phụ thuộc đều đạt yêu cầu. Ngoài hệ số $\mathrm{R}^{2}$ để đánh giá cấu trúc nội sinh, sự thay đổi của giá trị $\mathrm{R}^{2}$ khi một biến ngoại sinh bị loại bỏ cũng dùng để đánh giá biến bị loại bỏ đó có tầm quan trọng như thế nào đối với biến nội sinh. Chỉ số này gọi là hệ số tác động $\mathrm{f}^{2}$. Các giá trị $f^{2}$ ứng với $0.02,0.15$, và 0.35 , tương ứng với các trị tác động nhỏ, trung bình và lớn của biến ngoại sinh (Cohen, 2013). Nếu $f^{2}<0.02$ thì xem như không có tác động. $\mathrm{f}_{\mathrm{AT}-\mathrm{AA}}=$ 0,02 và $\mathrm{f}^{2}{ }_{\mathrm{PE}->\mathrm{AT}}=0,13$ là tác động nhỏ; còn các mối quan hệ còn lại thì $\mathrm{f}^{2}$ từ 0,181 đến 0,327 , do đó biến các biến ngoại sinh đóng vai trò trung bình trong tác động đến biến nội sinh. Cuối cùng, nghiên cứu kiểm tra khả năng dự báo của mô hình qua chỉ số $\mathrm{Q}^{2}$ cho thấy tất cả các giá trị $\mathrm{Q}^{2}$ đều $>0$. Điều này khẳng định khả năng dự báo cao của các biến ngoại sinh cho các biến nội sinh trong mô hình (Stone, 1974).

Như mong đợi, các hệ số đường dẫn giữa các cấu trúc trong mô hình ở bảng 5 đều có ý nghĩa với mức ý nghĩa $1 \%$. Bên cạnh đó, kết quả kiểm định Bootstrap cũng cho thấy các hệ số này đều khác 0 . Như vậy, có thể kết luận các giả thuyết từ $\mathrm{H} 1$ đến $\mathrm{H} 7$ đều được ủng hộ về mặt dữ liệu.

Bảng 5: Kết quả mô hình PLS-SEM

\begin{tabular}{|l|l|r|r|r|c|}
\hline \multicolumn{2}{|c|}{ Giả thuyết } & \multicolumn{1}{c|}{ Beta } & \multicolumn{1}{c|}{ t-value } & $\begin{array}{c}\text { Khoảng giá trị } \\
\text { (Bootstrap) }\end{array}$ & Kết luận \\
\hline AT -> AA & H1 & 0,144 & $3,706^{* * *}$ & {$[0,068-0,221]$} & Ủng hộ \\
\hline PU -> AA & H2 & 0,529 & $14,124^{* * *}$ & {$[0,455-0,601]$} & Ung hộ \\
\hline PU -> AT & H3 & 0,487 & $13,642^{* * *}$ & {$[0,411-0,554]$} & Unng hộ \\
\hline PE -> AT & H4 & 0,306 & $8,555^{* * *}$ & {$[0,230-0,372]$} & Ủng hộ \\
\hline PE -> PU & H5 & 0,362 & $7,776^{* * *}$ & {$[0,266-0,447]$} & Ủng hộ \\
\hline IN -> PU & H6 & 0,407 & $8,763^{* * *}$ & {$[0,317-0,496]$} & Ủng hộ \\
\hline IN -> PE & H7 & 0,467 & $8,696^{* * *}$ & {$[0,357-0,568]$} & Úng hộ \\
\hline
\end{tabular}

Ghi chú: *** $p<0,001$

\section{THẢO LUẬN}

Các giả thuyết từ $\mathrm{H} 1$ đến $\mathrm{H} 5$ đã khẳng định lại sự phù hợp khi ứng dụng mô hình TAM để phân tích cho bối cảnh sử dụng ứng dụng di động như một công nghệ cho việc lựa chọn các dịch vụ (Featherman \& Fuller, 2003).

Trong nghiên cứu này, nhận thức sự hữu ích có vai trò quan trọng trong mối quan hệ với ý định sử dụng ứng dụng $(\mathrm{Beta}=0,529, \mathrm{t}$-value $=14,124)$ và thái độ đối với việc sử dụng ứng dụng (Beta $=0,487$, $\mathrm{t}$-value $=13,642$ ) của thế hệ $Z$; từ đó, dẫn đến sử ảnh hưởng tích cực của thái độ đối với ứng dụng di động đến ý định sử dụng ứng dụng di động để lựa chọn các dịch vụ khi du lịch của giới trẻ thế hệ $Z$ (Beta $=0,144, t-$ value $=3,706$ ). Điều này cũng tương đối dễ hiểu khi thế hệ $Z$ là một thế hệ gắn liền với chủ nghĩa thực dụng (Chicca \& Shellenbarger, 2018; Wood, 2013), nên họ sẽ dễ bị thuyết phục bởi những giá trị hữu dụng cho các hoạt động trong đời sống, chẳng hạn như sử dụng các ứng dụng từ điện thoại để đặt tour du lịch, phòng khách sạn, hoặc lựa chọn địa điểm du lịch. Sự tiện lợi mà một ứng dụng di động mang lại sẽ tạo ra thái độ tích cực cho các du khách thế hệ $Z$, từ đó dẫn đến ý định sử dụng ứng dụng cho việc tìm kiếm thông tin và thực hiện các giao dịch khi du lịch. Việc sử dụng ứng dụng di động để tìm kiếm thông tin khi du lịch cũng góp phần gia tăng hiệu quả của chuyến du lịch cho các du khách trẻ tuổi, họ có thể lựa chọn đúng những địa điểm phù hợp với bản thân họ. Do đó, những cảm nhận hữu ích của việc sử dụng ứng dụng di động khi đi du lịch sẽ là tiền đề tốt cho thái độ, và hành vi sử dụng của du khách. Ả̉nh hưởng tích cực của cảm nhận dễ sử dụng ứng dụng di động cũng tác động tích cực đến thái độ của người sử dụng (Beta = $0,306, \mathrm{t}$-value $=8,555)$ và cảm nhận hữu ích $(B e t a=0,529$, $\mathrm{t}$-value $=14,124)$. Đối với du khách thuộc thế hệ $\mathrm{Z}$, việc sử dụng công nghệ trong cuộc sống dường như không quá khăn, các cư dân thế hệ $\mathrm{Z}$ dễ dàng sử dụng các công nghệ mới trong các hoạt động thông thường của đời sống. Ngoài ra, họ có thể tìm hiểu hướng dẫn sử dụng ứng dụng từ Google, hoặc các trang web công nghệ mà bản thân quan tâm. Những tiện lợi trong việc học cách sử dụng, cũng như sử dụng các ứng dụng di động khi du lịch đã tạo ra thái độ tích cực về công nghệ, cũng như cảm nhận sự hữu ích khi sử dụng ứng dụng.

Tính cách tân của người dùng đang nhận được sự quan tâm của nhiều nhà nghiên cứu khi nghiên cứu về sự chấp nhận công nghệ, đặc biệt những nghiên cứu liên quan đến nhóm người tiêu dùng thế hệ $\mathrm{Z}$ (LiébanaCabanillas và cộng sự, 2020; Wood, 2013). Tính cách tân được miêu tả là một tiền đề tích cực thúc đẩy người sử dụng công nghệ nhanh chóng tiếp cận công nghệ mới, hiện đại (Rogers, 1962). Nghiên cứu này 
đã chỉ ra tính cách tân ảnh hưởng tích cực đến cả cảm nhận hữu ích (Beta $=0,407$, t-value $=8,763$ ), và cảm nhận tính dễ sử dụng (Beta $=0,467, \mathrm{t}$-value $=8,696)$. Đầu tiên, tính cách tân của người dùng sẽ thúc đẩy họ sử dụng các công nghệ mới để nâng cao chất lượng của cuộc sống, do đó, những người cách tân sẽ nhận ra rằng sử dụng ứng dụng di động sẽ giúp họ có một hành trình thú vị hơn khi đi du lịch ở những nơi mà họ chưa từng đến trước đây. Ngoài ra, tính cách tân sẽ làm cho du khách trở nên chủ động hơn trong việc tìm kiếm các ứng dụng giúp họ tối ưu hóa chuyến đi, chẳng hạn như khách sạn nào giá rẻ và chất lượng, hoặc quán ăn nào được đánh giá cao tại địa điểm du lịch cụ thể. Đối với một người cách tân, họ luôn sẵn sàng sử dụng công nghệ mới để trải nghiệm, dù cho công nghệ đó có khó sử dụng và có thể rủi ro nhưng họ sẽ tìm cách để chinh phục công nghệ đó. Những du khách thế hệ $Z$ sẽ tìm cách học để sử dụng các công nghệ nhằm phục vụ nhu cầu du lịch của bản thân, từ đó họ sẽ dễ dàng trong việc sử dụng ứng dụng trên chính thiết bị di động của họ. Khi khoa học kỹ thuật phát triển, các ứng dụng ngày càng có chức năng đa dạng và giao diện thân thiện với người dùng, do đó, những người dùng cách tân có thể tùy biến cho ứng dụng phù hợp với bản thân hơn thay vì sử dụng theo đúng giao diện hoặc chức năng được nhà sản xuất đề xuất.

\section{KẾT LUẦN}

Kết quả nghiên cứu đã góp phần khẳng định ý nghĩa của mô hình Chấp nhận công nghệ của Davis (1986) ở bối cảnh ý định sử dụng ứng dụng di động để lựa chọn các dịch vụ khi du lịch của giới trẻ thế hệ $Z$ trong thời kỳ chuyển đổi số. Kết quả nghiên cứu phù hợp với các nghiên cứu ứng dụng mô hình chấp nhận công nghệ TAM ở các lĩnh vực khác như chấp nhận sử dụng máy vi tính (Dinev và cộng sự, 2009), thương mại điện tử (Featherman \& Fuller, 2003), thương mại di động (Alsamydai, 2014), giáo dục (Khoa và cộng sư, 2020). Đồng thời, nghiên cứu cũng đã đóng góp trong việc mở rộng mô hình TAM. So với các nghiên cứu trước đây, nghiên cứu này đã bổ sung thêm yếu tố tính cách tân của người dùng dựa trên phân loại của Rogers (1962). Tính cách tân của người dùng được chứng minh là ảnh hưởng tích cực đến các yếu tố chính trong mô hình TAM như cảm nhận sự hữu ích và cảm nhận tính dễ sử dụng của một sản phẩm công nghệ. Các doanh nghiệp cung cấp các dịch vụ du lịch cần có sự kết hợp giữa tiếp thị truyền thống và tiếp thị công nghệ, nghĩa là sử dụng hình thức tiếp thị qua phương tiện kỹ thuật số như điện thoại di động để nâng cao hiệu quả kinh doanh. Đầu tiên, doanh nghiệp phải tạo tiền đề cho ý định chấp nhận sử dụng ứng dụng di động cho bằng cách tạo ra thái độ tích cực cho du khách. Những hoạt động truyền thông về tính tiện lợi cũng như những ưu đãi khi sử dụng ứng dụng để đặt phòng hoặc dịch vụ sẽ gia tăng cảm xúc tích cực của du khách. Đặc biệt, đối với khách hàng thuộc thế hệ $Z$, những người thuộc về thế hệ công nghệ, doanh nghiệp cần phải tạo ra những nội dung quảng cáo mang tính gắn kết với đặc điểm và nhu cầu của họ nhu "sành điệu khi đặt dịch vụ qua ứng dụng di động", hoặc "chứng tỏ bản thân khi dùng ứng dụng". Doanh nghiệp cũng cần tăng cường các tính năng cho ứng dụng, như ví tiền điện tử, quản lý và lưu trữ thông tin những điểm đến được yêu thích; điều này sẽ góp phần nâng cao sự hữu dụng của ứng dụng trên di động. Ngoài ra, tốc độ xử lý các giao dịch trên ứng dụng cũng phải nhanh và chính xác nhằm tạo ra hiệu quả cho quá trình du lịch của du khách. Để tạo ra nhận thức về tính dễ sử dụng cho ứng dụng, doanh nghiệp nên chú trọng vào việc lập trình và thiết kế giao diện sao cho thân thiện với người dùng. Đồng thời, doanh nghiệp cũng cần phải có các hướng dẫn cụ thể trong ứng dụng, hoặc trên website để du khách có thể tự học cách sử dụng. Trong khi hầu hết các doanh nghiệp có ý định cung cấp các sản phẩm và dịch vụ tốt nhất cho khách hàng, cách tiểp cận của họ thường bị thúc đẩy bởi niềm tin, tư duy và ý tưởng của chính họ về những gì khách hàng cần và cách cư xử của mọi người. Mặc dù việc nhìn thế giới qua góc nhìn của chính mình là điều hoàn toàn tự nhiên, nhưng đó là vấn đề vì các tổ chức có khả năng phát triển một giải pháp phù hợp với khách hàng. Do đó, các doanh nghiệp cần phát triển sự đồng cảm đích thực đối với khách hàng. Họ nên gần gũi và quan sát những khách hàng đang đổi mới để hiểu nhu cầu, động cơ và hành vi thực tế của họ nhằm tìm kiếm các giải pháp khả thi, có giá trị và bền vững hơn. Các việc làm cụ thể để hiểu được khách hàng đó là thông qua nghiên cứu thị trường, hoặc tổ chức các buổi tiếp xúc với khách hàng.

Mặc dù đã nỗ lực tối đa nhằm đặt được mục tiêu nghiên cứu, tuy nhiên, nghiên cứu này cũng còn một số hạn chế nhất định. Đầu tiên, nghiên cứu phát triển mô hình nghiên cứu dựa trên lý thuyết TAM căn bản, điều này có thể dẫn đến hạn chế tính cập nhật của nghiên cứu. Do đó, những nghiên cứu sau có thể phát triển một nghiên cứu mới dựa trên các lý thuyết chấp nhận công nghệ mới nhất, như TAM2, TAM3. Hơn nữa, mặc dù nghiên cứu tập trung vào đối tượng du khách ở thế hệ $Z$ nhằm làm rõ mục tiêu nghiên cứu, nhưng việc mở rộng nghiên cứu nhằm mục đích so sánh giữa thế hệ $\mathrm{Y}$ và thế hệ $\mathrm{Z}$ sẽ có thể mang lại các 
kết luận thú vị hơn. Bên cạnh đó, nghiên cứu có thể tiến hành kiểm định mối quan hệ giữa các yếu tố trong mô hình $\mathrm{TAM}$, chẳng hạn mối quan hệ giữa cảm nhận dễ sử dụng và ý định sử dụng công nghệ như trong nghiên cứu của Bae và Han (2020).

\section{TÀI LIỆU THAM KHẢO}

Agag, G., \& El-Masry, A. A. (2016). Understanding consumer intention to participate in online travel community and effects on consumer intention to purchase travel online and WOM: An integration of innovation diffusion theory and TAM with trust. Computers in Human Behavior, 60, 97-111. doi: 10.1016/j.chb.2016.02.038

Agarwal, R. (2000). Individual acceptance of information technologies. In Framing the domains of IT management. (pp. 85-104). Cincinnati, OH: Pinnaflex Education Resources.

Al-Rahmi, W. M., Yahaya, N., Aldraiweesh, A. A., Alamri, M. M., Aljarboa, N. A., Alturki, U., \& Aljeraiwi, A. A. (2019). Integrating technology acceptance model with innovation diffusion theory: An empirical investigation on students' intention to use E-learning systems. IEEE Access, 7, 26797-26809.

Alsamydai, M. J. (2014). Adaptation of the technology acceptance model (TAM) to the use of mobile banking services. international review of management and business research, 3(4), 2039.

Arif, I., Afshan, S., \& Sharif, A. (2016). Resistance to Adopt Mobile Banking in a Developing Country: Evidence from Modified TAM. Journal of Finance \& Economic Research, 1(1), 25-42. doi: 10.20547/jfer1601104

Ayar, B., GÜVendiK, Ö., \& AydoĞAn, S. (2019). Innovative Consumers of the Digital Age: Opinion Seeking on Instagram. Business \& Management Studies: An International Journal, 7(2), 609-628. doi: 10.15295/bmij.v7i2.966

Bae, S. Y., \& Han, J. H. (2020). Considering Cultural Consonance in Trustworthiness of Online Hotel Reviews among Generation Y for Sustainable Tourism: An Extended TAM Model. Sustainability, 12(7), 2942.

Bandura, A. (1982). Self-efficacy mechanism in human agency. American Psychologist, 37(2), 122.

Braun, S. (2019). Technology and interpreting. In M. O'Hagan (Ed.), The Routledge Handbook of Translation and Technology. New York: Routledge.

Chandio, F., Burfat, F., Abro, A., \& Naqvi, H. (2017). Citizens' acceptance and usage of Electronic-Government services: A conceptual model of trust and technological factors. Sindh University Research Journal-SURJ (Science Series), 49(3), 665-668.

Chicca, J., \& Shellenbarger, T. (2018). Connecting with Generation Z: Approaches in nursing education. Teaching and Learning in Nursing, 13(3), 180-184.

Chuttur, M. Y. (2009). Overview of the technology acceptance model: Origins, developments and future directions. Working Papers on Information Systems, 9(37), 9-37.

Cohen, J. (2013). Statistical Power Analysis for the Behavioral Sciences. Hoboken: Taylor and Francis. doi: $10.4324 / 9780203771587$

Davis, F. D. (1986). A technology acceptance model for empirically testing new end-user information systems: Theory and results. (Doctor of Philosophy). Massachusetts Institute of Technology, USA. Retrieved from http://hdl.handle.net/1721.1/15192

Davis, F. D. (1989). Perceived Usefulness, Perceived Ease of Use, and User Acceptance of Information Technology. MIS Quarterly, 13(3), 319-340. doi: 10.2307/249008

Davis, F. D., Bagozzi, R. P., \& Warshaw, P. R. (1989). User acceptance of computer technology: a comparison of two theoretical models. Management Science, 35(8), 982-1003.

Dinev, T., Goo, J., Hu, Q., \& Nam, K. (2009). User behaviour towards protective information technologies: the role of national cultural differences. Information Systems Journal, 19(4), 391-412.

Featherman, M., \& Fuller, M. (2003). Applying TAM to e-services adoption: the moderating role of perceived risk. Paper presented at the Proceedings of the 36th Hawaii International Conference on System Sciences, Big Island, HI, USA. doi: 10.1109/HICSS.2003.1174433

GlobalWeb Index. (2016). Data Point: Gen Z Spends Over 3.5 Hrs On Mobiles Daily. Retrieved from https://arabianmarketer.ae/data-point-gen-z-spends-over-3-5-hrs-on-mobiles-daily/

Hair, J. F., Hauff, S., Hult, G. T. M., Richter, N. F., Ringle, C. M., \& Sarstedt, M. (2017). Partial Least Squares Strukturgleichungsmodellierung. London: Sage publications. doi: 10.15358/9783800653614

Hoa, L. T. K., \& Khoa, B. T. (2021). Động lực nghiên cứu khoa học của giảng viên: Góc nhìn lý thuyết nhu cầu mở rộng của Maslow. Tạp chi Khoa học và Công nghệ - IUH, 46(04), 235-248. doi: 10.46242/jst-iuh.v46i04.706

Khoa, B. T. (2020a). The Impact of the Personal Data Disclosure's Tradeoff on the Trust and Attitude Loyalty in Mobile Banking Services. Journal of Promotion Management, 27(4), 585-608. doi: 10.1080/10496491.2020.1838028 Khoa, B. T. (2020b). The role of Mobile Skillfulness and User Innovation toward Electronic Wallet Acceptance in the Digital Transformation Era. Paper presented at the 2020 International Conference on Information Technology Systems and Innovation (ICITSI), Bandung - Padang, Indonesia. doi: 10.1109/ICITSI50517.2020.9264967 
Khoa, B. T., Nguyen, H. M., Tran, N. V. H., \& Nguyen, B. H. (2020). Lecturers' adoption to use the online Learning Management System (LMS): Empirical evidence from TAM2 model for Vietnam. Journal of Science Hcmcou Economics \& Business Administration, 10(1), 3-17. doi: 10.46223/HCMCOUJS.econ.en.10.1.216.2020

Khoa, B. T., \& Nguyễn, M. H. (2020). Nghiên cứu sự đánh đổi giữa lợi ích và chi phí khi sử dụng dịch vụ trực tuyến: Trường hợp thương mại di động tại Việt Nam. Journal of Science and Technology - IUH, 41(05), 141-155. doi: 10.46242/jst-iuh.v41i05.271

Lee, J. M., \& Wei, L. (2018). Gen Z Is Set to Outnumber Millennials Within a Year. Retrieved from https://www.bloomberg.com/news/articles/2018-08-20/gen-z-to-outnumber-millennials-within-a-year-demographic$\underline{\text { trends }}$

Lee, K.-W., Tsai, M.-T., \& Lanting, M. C. L. (2011). From marketplace to marketspace: Investigating the consumer switch to online banking. Electronic Commerce Research and Applications, 10(1), 115-125.

Liébana-Cabanillas, F., García-Maroto, I., Muñoz-Leiva, F., \& Ramos-de-Luna, I. (2020). Mobile Payment Adoption in the Age of Digital Transformation: The Case of Apple Pay. Sustainability, 12(13), 5443.

Lin, S., Zimmer, J. C., \& Lee, V. (2014). Decoupling software from hardware in technology acceptance research. Journal of Computer Information Systems, 54(2), 77-86.

Luan, W. S., \& Teo, T. (2009). Investigating the technology acceptance among student teachers in Malaysia: An application of the technology acceptance model (TAM). The Asia-Pacific Education Researcher, 18(2), 261-272.

Munoz-Leiva, F., Climent-Climent, S., \& Liébana-Cabanillas, F. (2017). Determinants of intention to use the mobile banking apps: An extension of the classic TAM model. Spanish Journal of Marketing-ESIC, 21(1), 25-38.

Napitupulu, D. (2017). A conceptual model of e-government adoption in Indonesia. International Journal on Advanced Science, Engineering and Information Technology, 7(4), 1471-1478.

Nunnally, J. C., \& Bernstein, I. (1994). The assessment of reliability. Psychometric theory, 3(1), 248-292.

Radulović, L. (2013). The role and potential of Global Distribution System Amadeus for Tourism development at the global level. The European Journal of Applied Economics, 10(1), 28-38.

Ramos de Luna, I., Montoro-Ríos, F., Martínez-Fiestas, M., \& Casado-Aranda, L.-A. (2020). Analysis of a Mobile Payment Scenario: Key Issues and Perspectives. In Impact of Mobile Services on Business Development and ECommerce (pp. 22-47). Pennsylvania, USA: IGI Global.

Rogers, E. M. (1962). Diffusion of innovations. New York: Free Press.

Santini, F. d. O., Ladeira, W. J., Sampaio, C. H., \& Boeira, J. P. (2020). The Effects of Sales Promotions on Mobile Banking a Cross-Cultural Study. Journal of Promotion Management, 26(3), 350-371. doi: $10.1080 / 10496491.2019 .1699622$

Stone, M. (1974). Cross-validatory choice and assessment of statistical predictions. Journal of the Royal Statistical Society: Series B (Methodological), 36(2), 111-133.

Syed-Abdul, S., Malwade, S., Nursetyo, A. A., Sood, M., Bhatia, M., Barsasella, D., . . Raja, M. (2019). Virtual reality among the elderly: a usefulness and acceptance study from Taiwan. BMC Geriatrics, 19(1), 223.

Taib, S. M., De Coster, R., \& Nyamu, J. (2016). Innovation diffusion of wearable mobile computing: Pervasive computing perspective. Paper presented at the 2016 International Conference on Information Society (i-Society).

Torres, R., \& Gerhart, N. (2017). Mobile Proximity Usage Behaviors Based on User Characteristics. Journal of Computer Information Systems, 59(2), 161-170. doi: 10.1080/08874417.2017.1320954

Venkatesh, V., \& Davis, F. D. (2000). A theoretical extension of the technology acceptance model: Four longitudinal field studies. Management Science, 46(2), 186-204. doi: https://doi.org/10.1287/mnsc.46.2.186.11926

Vipin, J. (2016). How Mobile App Solutions Has Transformed The Travel Industry. Retrieved from https://www.digitaldoughnut.com/articles/2016/september/how-mobile-app-solutions-has-transformed-travel-in Wood, S. (2013). Generation Z as consumers: trends and innovation. Institute for Emerging Issues: NC State University, 1-3.

World Tourism Organization. (2016). Global Report on The Power of Youth Travel Retrieved from Madrid: https://www.wysetc.org/wp-content/uploads/2016/03/Global-Report_Power-of-Youth-Travel_2016.pdf

Zhang, P., Aikman, S. N., \& Sun, H. (2008). Two Types of Attitudes in ICT Acceptance and Use. International Journal of Human-Computer Interaction, 24(7), 628-648. doi: 10.1080/10447310802335482

Ngày nhận bài: 11/10/2020

Ngày chấp nhận đăng: 05/03/2021 